\title{
Introductory editorial to the special issue on systems medicine for the delivery of better healthcare services
}

\author{
Athos Antoniades ${ }^{1} \cdot$ Constantinos S. Pattichis $^{2} \cdot$ Christos N. Schizas $^{2}$. \\ Panagiotis D. Bamidis ${ }^{3}$
}

Received: 8 December 2016 / Accepted: 15 December 2016 / Published online: 23 December 2016

(C) IUPESM and Springer-Verlag Berlin Heidelberg 2016

Medical and Biological Engineering and Computing cover complementary disciplines that hold great promise for the advancement of research and development in complex medical and biological systems. Research and development in these areas are impacting the science and technology by advancing fundamental concepts in translational medicine, by helping us understand human physiology and function at multiple levels, by improving tools and techniques for the detection, prevention and treatment of disease. This special issue is based on a selected number of papers under the main theme "Systems Medicine for the Delivery of Better Healthcare Services" that were presented in the XIV Mediterranean Conference on Medical and Biological Engineering and Computing Medicon 2016, March 31st-April 2nd, 2016, that was organised in Cyprus (http://medicon2016.org/). The goal of Medicon 2016 was to provide updated information on the state of the art on Medical and Biological Engineering

This article is part of the Topical collection on Systems Medicine

Athos Antoniades

athos.antoniades@stremble.com

Constantinos S. Pattichis

cpattichis@gmail.com; pattichi@ucy.ac.cy

Christos N. Schizas

schizas@cs.ucy.ac.cy

Panagiotis D. Bamidis

bamidis@med.auth.gr

Stremble Ventures LTD, Limassol, Cyprus

2 Department of Computer Science, University of Cyprus, Nicosia, Cyprus

3 Faculty of Medicine, Aristotle University of Thessaloniki, Thessaloniki, Greece and provided a common platform for the cross fertilization of ideas, and helped shaping the knowledge and scientific achievements by bridging complementary disciplines into an interactive and attractive forum under the conference theme that was the same as the theme of the special issue.

A total of 6 editorial papers and 14 regular papers are published in this special issue under the following four thematic areas: Editorial, Sensing and Diagnosing Systems, Imaging Systems, Medical Informatics Systems and Bionformatics. The Editorial section is highlighted with the position papers presented in the corresponding thematic session on Systems Medicine in Medicon 2016. It includes the editorial on The Evolution of Medical Care: From the Beginnings to Personalized Medicine by Prof Demetris Koutsouris, National Technical University of Athens, Greece; the position papers on Systems Medicine for the Delivery of Better Healthcare Services - IFMBE Perspective, IOMP Perspective and IUPESM perspective given by Prof James Goh, President, IFMBE, Prof Slavik Tabakov, President, IOMP and Prof Kin Yin Cheung President, IUPESM, respectively; the editorial on Cognitive Computing for Supporting eHealth by Prof Christos N. Schizas, University of Cyprus, Cyprus.

The section on Sensing and Diagnosing Systems has 6 papers. The paper by Manis et al. describes the variation of fetal heart rhythm towards delivery. The paper by Vala et al. carries out a comparison of simple algorithms for estimating respiration rate from electrical impedance pneumography signals in wearable devices. The paper by Karkanis and coworkers covers the classification of neuromuscular disorders based on EMG wavelet analysis. IoT of indoor analytics of active and healthy ageing is investigated by Konstantinidis et al. Furthermore, indoor localisation through object detection utilising a single wearable camera is investigated by Shewell and coworers. The paper by Laufer and coworkers 
proposes an lung mechanics airway resistance dynamic elastance model in support of the assessment of the mechanical ventilation therapy of patients with acute respiratory distress syndrome.

The section on imaging systems has 1 paper. The paper by Giannini et al. describes the specificity improvement of a CAD system for multiparametric MR prostate cancer using texture features and artificial neural networks.

The section on medical informatics systems has 4 papers. Reihs and coworkers present a system facilitating the automatic classification of histopathological diagnoses for building a large scale tissue catalogue. The second paper is also from the same group, proposing the evaluation of existing solutions to an allinclusive package for biobanks. The third paper by Stylianou and Talias present a discussion on the big data challenges in healthcare. The fourth paper by Lejla and coworkers present a software solution for the inspection process of medical devices in healthcare institutions in Bosnia and Herzegovina.

The section on Bionformatics has 3 papers. The paper by Sfakianakis and coworkers carries out exploratory analysis of local gene groups in breast cancer guided by biological networks. The paper by Chatziioannou investigates an RNA-sequence analysis from non-small cell lung cancer biopsies, and the paper by Pashaei and coworkers investigates random forest based analysis in splice site prediction of the human genome.
The goal of this special issue is to present a snapshot of original and relevant contributions covering the area of systems medicine for the delivery of better healthcare services. It is hoped that the proposed technologies and systems can result in improved disease management and treatment at the point of need, reduced hospitalization, and the associated economic burden, offering a better quality of life to the patient.

Acknowledgements We would like to thank all the contributors of the special issue and all the reviewers who put a great deal of their time and effort, under very tight deadlines, in order to help us review all the papers and put the special issue together. Moreover, we would like to thank Jennielyn Flores at the journal editorial office for her patience and help in materialising this special issue. Last but not least we would like to express our sincere thanks to the journal Editor, Luis Kun for his wisdom and guidance throughout the course of this special issue.

\section{Compliance with ethical standards}

Conflict of interest No conflict of interest.

Funding There is no funding source.

Ethical approval This article does not contain any studies with human participants or animals performed by any of the authors.

Informed consent Not applicable. 